\title{
Image analysis of small animal feeding behavior
}

\author{
MARC ROWLEY \\ University of Maryland, Baltimore County, Maryland \\ JOE STITT \\ Pennsylvania State University, State College, Pennsylvania \\ and \\ FRANK HANSON \\ University of Maryland, Baltimore County, Maryland
}

\begin{abstract}
The optimal design of behavioral experiments includes measures to minimize observer bias while maximizing researcher efficiency. To this end, we have developed a reliable, autonomous monitor of animal feeding behavior. Previous studies of caterpillar (Manduca sexta) feeding have relied on human observation for visual evaluation of food consumption at specific time points over the course of several hours. This method is eliminated by our new behavior rig, which collects data automatically. Individual animals are monitored via CCD cameras activated in sequence by a computer. Images are obtained at preset time intervals using a frame grabber to capture still pictures from the cameras. Subsequently, images are analyzed using software written in MatLab to determine food selection and quantify consumption.
\end{abstract}

Feeding is arguably one of the most important of all animal behaviors. Without the ability to select appropriate foods, an animal could not survive. That behavior and its underlying physiology have been investigated in insects because of their relative simplicity and economic importance. Our study animal is the tobacco hornworm, larval Manduca sexta, which is a commonly used model system for studying insect behavior and physiology. Our research is focused on food selection behavior, which is controlled largely by the animal's sense of taste (Schoonhoven \& van Loon, 2002).

Feeding behavior is assayed using the disk test, which was developed in the mid-1960s (Jermy, Hanson, \& Dethier, 1968) and is commonly used for this type of research (de Boer, 1993; de Boer \& Hanson, 1984). This test is easy to perform, produces repeatable data, is statistically sound (Lockwood, 1998), and has an interindividual standard error of $5 \%$ or less. With this standard error and a sample size of 20 individuals, we are able to detect a 5\% difference in consumption between treatment groups. The test format is flexible: Two- to six-choice tests that compare across food types and no-choice tests that compare feeding rates across animals can be done.

The original test involved affixing leaf disks in a behavioral arena with pins pushed into a paraffin wax substrate.

The authors thank George Johnson for electronics assistance and Chuck Sperandeo for design assistance and construction of the arena trays and rig equipment. We also thank Tim Ford for his assistance with the figures. Correspondence concerning this article should be addressed to M. Rowley, University of Maryland, Department of Biological Sciences, 1000 Hilltop Circle, Baltimore, MD 21250 (e-mail: mrowle3 @ umbc.edu)
Consumption of the disks was determined by visual estimation of the percentage of each disk that remained. These observations were normally made every hour for $6-$ $12 \mathrm{~h}$. Thus, the sources of error included missed samples as well as observer estimation error and bias. To remedy these problems and increase efficiency, we have developed a behavior rig in which the data collection process is completely automated, thus permitting unattended trials.

\section{Hardware}

The Arena. Animals are placed in a behavioral arena containing six equidistant food disks (of either leaves or glass fiber filter paper spotted with plant chemicals) $1.6 \mathrm{~cm}$ in diameter located around the perimeter (Figure 1A). These food disks are held in place by a pin placed through the disk and into a small tubular standoff (made of HPLC tubing; Upchurch Scientific 1533) that keeps the disk $1 \mathrm{~cm}$ above the floor of the arena (Figure 1B). A short section of Tygon tubing (Fisher Scientific 14-169-1B) is placed around the standoff to form a better seal for the pinhead/standoff assembly, thus decreasing the amount of fluid wicked away from the disk and along the pin shaft. A plastic grid allows the animals to grip the floor.

To avoid desiccation of the food disks, wetted filter paper is placed on the arena floor as a source of humidity. To prevent the insects from feeding on this source of humidity, we use creped, wet-strengthened filter paper (Fisher Scientific 09-790-12C). The entire arena is then covered by an optically flat petri dish to contain the animal and prevent evaporation while still permitting undistorted image collection.

The video system. The video system is constructed as is shown in Figure 2. Small CCD cameras (Supercircuits 
A
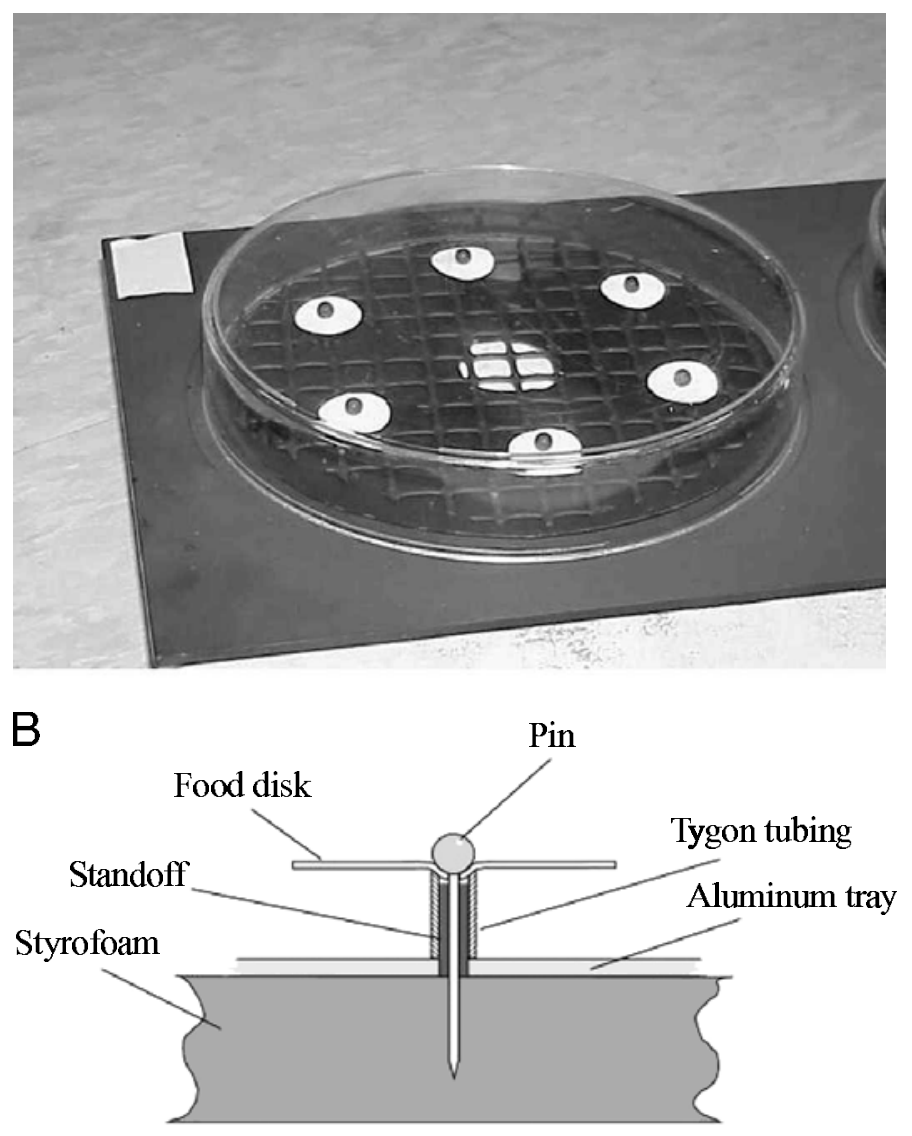

Figure 1. The behavioralarena. (A) Six filter paper disks are arranged equidistant from each other and are held in place with pins. Test chemicals are placed on disks at the start of the experiment in an ABABAB fashion for a two-choice test, in an $\mathrm{ABCABC}$ fashion for a three-choice test, and so on. An animal is placed in the center of the arena, selects a disk, and begins feeding. (B) Details of disk and pin assembly.

PC100X), each viewing a single arena, are arranged in an array. These cameras are activated by software running on a Macintosh PowerPC 7500 computer with a digital I/O card (National Instruments PCI-DIO-96) operating a bank of DPST reed relays that both sequentially activate individual cameras and route their video signals to the frame grabber (Computer Eyes/RT Video Frame Grabber). After a 5-sec warm-up for the cameras, the frame grabber is instructed to acquire an image. These grayscale images are then saved to the computer's hard drive for later analysis.

Lighting within the rig is provided by a set of rope lights (Good Tidings, Baltimore, MD). These lights are run through translucent glass fiber tubes to diffuse the light. A dimmer switch controls light intensity. This system provides an inexpensive source of soft illumination adequate for the CCD cameras.

\section{Software}

Image acquisition. Image acquisition is controlled by software written in C. Through an ASCII text file, the user specifies the number of images to acquire and the time intervals between images. A typical experiment of ours collects 1 image taken at the beginning of the run (Time 0 ), 2 images at 15 -min intervals, then 6 at 30-min. intervals, and finally 10 images at $1-\mathrm{h}$ intervals. This results in a total collection of 19 images for each arena over a period of $13.5 \mathrm{~h}$.

Image analysis. Once the experiment is over, the collected images are moved to a faster computer (Macintosh G4) for image analysis. The analysis programs are written in MatLab (MathWorks) and are thus portable to any computing platform for which MatLab is available, such as Microsoft Windows and Unix. In general terms, the software is designed to organize the images into 3-D arrays, remove the nondata portions of these images, and determine consumption. These tasks are accomplished using a series of three MatLab programs: genIA, reduceIA, and calcArea.

Generation of the image array (genIA). A large number of images per experiment, typically 19 images per an- 


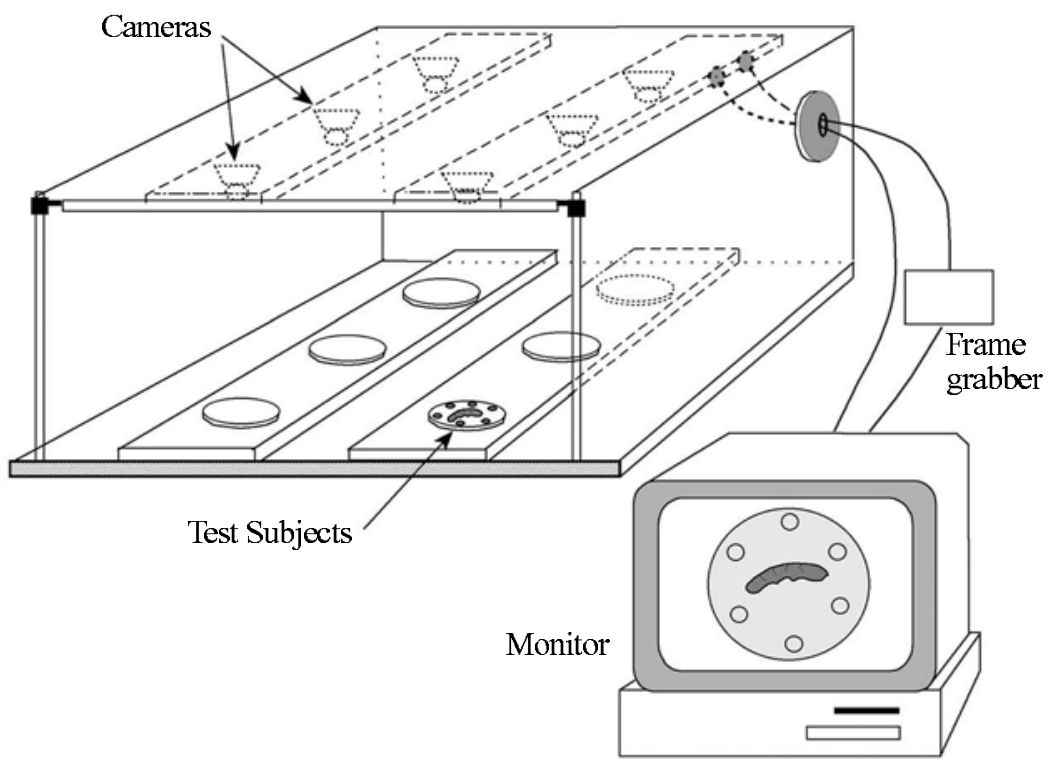

Figure 2. The behavior rig. Strips of CCD cameras are positioned above the behavior arenas on a frame. Each camera monitors a single arena. A computer with a digital I/O card activates the cameras and frame grabber in sequence, then stores the frame on its hard drive.

imal, are produced at $1.1 \mathrm{MB}$ per image. The program genIA combines all 19 images into one file by creating a 3-D array of the image data for each animal (Figure 3). These arrays are saved as .MAT files, significantly decreasing the time required to load them into the MatLab workspace.

Reduction of image to the area of interest (reduceIA). Our second program reduces the images to only the food disks and a small perimeter of background. These food disks are identified on the arena image by moving cross hairs with the mouse and clicking at the approximate center of each disk. This is done only for the first trial to establish the coordinates of the disk centers so that data collection can be restricted to a window in an area only slightly larger than the actual food disk (Figure 3 ).

This process of manually locating the food disk is flexible and robust for various food colors and lighting conditions. We have also used automated centroid-finding algorithms to locate disk centers, but these are less flexible and more sensitive to lighting conditions and contrast.

The new files created are 3-D arrays of disk images of almost exclusively data-containing regions. This reduces the file size to about 1 megabyte (from 20 megabytes) for each animal and speeds subsequent processing.

Calculation of the area consumed (calcArea). The third program extracts the consumption data, which is pro-

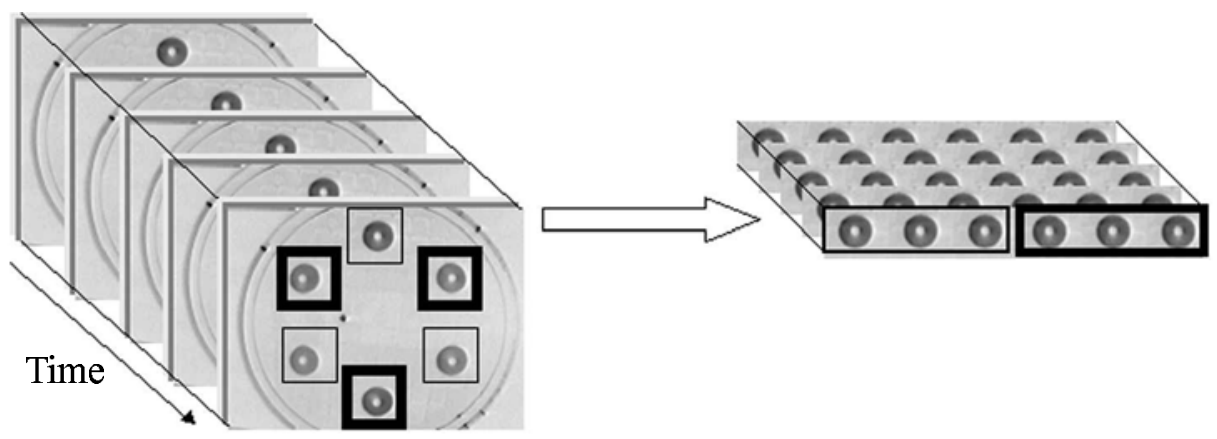

Figure 3. Image array reduction. All the raw images from a single arena-that is, one from each sample time-are imported into MatLab, arranged in a 3-D matrix, and saved as one file (genIA). Thus, a separate image file is generated for each arena in the experiment. Image array files are then reduced in size by cutting out "windows" around each of the food items (reduceIA). These windows are arranged as strips of images in a 3-D matrix containing the disk area with a small amount of background. 
A

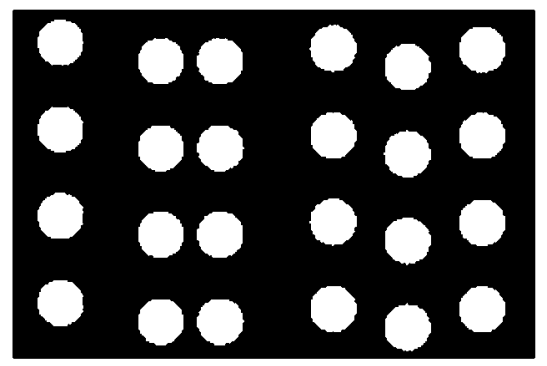

B

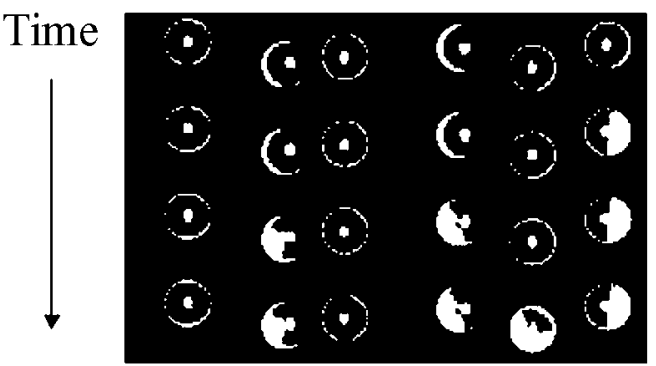

Figure 4. Measuring consumption with binary images. (A) Positive binary images of Trial 1 are created by the software for each arena. (B) These images are then overlaid (Boolean AND) with negative binary images of each subsequent trial. As more consumption occurs with time, more white space is revealed and measured.

portional to the disk area that has disappeared over time. The grayscale disk images produced in reduceIA are processed by a gray-level thresholding routine that converts them to binary (black and white) images. Those of the first (preconsumption) trial are saved as positive images - that is, the food disks are white and the surrounding background and pinhead are black (Figure 4A). Images from subsequent trials are negative images (food disks black, background white) that are overlaid upon the first trial (positive images) using the Boolean AND function such that any part of the disk not eaten will appear black. As more of the food disks are consumed, more white space appears (Figure 4B). Consumption is measured by counting white pixels and dividing by the original pixel count to yield the percentage of each disk consumed. Data is output as an array in a .MAT file or text file, or is printed as a table (Table 1).

\section{Sources of Error}

Two sources of error are inherent to our system. The first source of error is the insect itself. Occasionally, the insect is present in the area of the food disk during the acquisition of an image, which results in a calculated negative consumption of that disk at that time point. Since consumption is calculated independently at each time point, this will not affect subsequent readings. A survey of our data shows that out of 473 trials $(8,987$ time points) an insect was found in the area of the food disk $4 \%$ of the time (369 time points). This averages to 1 disrupted image (out of 19) per animal. These aberrations are easily seen when we do the consumption-versus-time plots following data analysis.

The second source of error is from the cameras. Our CCD cameras continuously adjust to the ambient light intensity, resulting in small shifts in recorded gray-level values at the pixel level. This is evident in the images as noise in the grayer areas of the food disk (edges, shadows). The gray-level thresholding step in calcArea eliminates most of this noise except near threshold. On the basis of variability of repeated measures in insect-absent trials $(N=15)$, such noise accounts for a $2 \%$ variation between consumption readings.
A visual inspection of images in calcArea permits identification of events such as these. Suspect data points can be flagged by the user for later consideration during statistical analysis.

\section{Applications}

Although we have developed this system to record and analyze caterpillar feeding behavior, it can easily be applied to analyze the feeding behavior of any small animal. The system requires that the food items be two-dimensional and is most effective in comparisons over time. The parameters can easily be changed to accommodate arenas of various sizes and numbers of food items. Having the user locate these food items through the reduceIA interface also gives the researcher complete freedom in placing these items throughout the arena. Since the data are out-

Table 1

Sample Data Output for Dish 1

\begin{tabular}{rrr}
\hline Pass & Group A & Group B \\
\hline 1 & 0.00 & 0.00 \\
2 & 3.86 & 5.83 \\
3 & 2.87 & 11.18 \\
4 & 24.76 & 89.95 \\
5 & 87.93 & 108.29 \\
6 & 100.29 & 167.29 \\
7 & 112.12 & 208.47 \\
8 & 118.52 & 235.76 \\
9 & 134.11 & 283.03 \\
10 & 210.53 & 290.24 \\
11 & 247.98 & 289.82 \\
12 & 253.62 & 288.35 \\
13 & 266.64 & 286.36 \\
14 & 270.46 & 294.92 \\
15 & 270.39 & 295.21 \\
16 & 268.72 & 286.70 \\
17 & 267.81 & 295.08 \\
18 & 267.33 & 295.30 \\
19 & 266.07 & 294.55 \\
\hline
\end{tabular}

Note-This table is an example of the normal output of calcArea for a deterrent (control disks are preferred over experimental disks). Group A is the sum of three experimental food disks and Group B is the sum of the controls. Pass number is equivalent to time point number. Note the "negative feeding" at pass 16 . 
put as both a MatLab array file and a text file, data analysis can be done using either MatLab or another software package.

\section{Availability}

This software and the detailed hardware specifications can be obtained by contacting M. Rowley at mrowle3@ umbc.edu. An earlier version of this system was described in Stitt, Gaumond, Frazier, and Hanson (1998).

\section{REFERENCES}

DE Boer, G. (1993). Plasticity in food preference and diet-induced differential weighting of chemosensory information in larval Manduca sexta. Journal of Insect Physiology, 39, 17-24.

DE Boer, G., \& Hanson, F. E. (1984). Foodplant selection and induction of feeding preference among host and non-host plants in larvae of the tobacco hornworm Manduca sexta. Entomologia Experimentalis et Applicata, 35, 177-193.

Jermy, T., Hanson, F. E. \& Dethier, V. G. (1968). Induction of specific food preference in lepidopterous larvae. Entomologia Experimentalis et Applicata, 11, 211-230.

Lockwood, J. R, III (1998). On the statistical analysis of multiplechoice feeding preference experiments. Oecologia, 116, 475-481 Schoonhoven, L. M., \& VAn Loon, J. J. A. (2002). An inventory of taste in caterpillars: Each species its own key. Acta Zoologica Academiae Scientiarum Hungaricae, 48(Suppl. 1), 215-263.

Stitt, J. P., Gaumond, R. P., Frazier, J. L., \& Hanson, F. E. (1998) Automated analysis of feeding behavior in small animals. In Proceed ings of the IEEE 24th Annual Northeast Bioengineering Conference (pp. 120-123). Piscataway, NJ: IEEE Press.

(Manuscript received September 19, 2002; revision accepted for publication May 4, 2003. .) 